\title{
The development of an IoT Infrastructure to Perform Behavioural Change Towards Low Energy Habits
}

\author{
Alfonso P. Ramallo-González, Aurora González-Vidal, Pedro J. Fernandez-Ruiz, Antonio \\ Skarmeta-Gómez \\ Universidad de Murcia, Murcia, Spain
}

\begin{abstract}
Building simulation allows to understand on a very precise manner the way in which the energy flows are occurring within a building. This, apart from being necessary to evaluate the energy performance, offers the possibility of tackling several aspects of the building operation. In most buildings, occupants are capable of modifying operation parameters such as switching off the lights, or changing the set point temperatures. A poor selection of these operational parameters may lead to a larger energy consumption that one may expect. Although some buildings have amperimetric clamps and other sensors that can inform about energy consumption, it is practically impossible to measure all the energy flows that can be given by a model. Making use of this, a building has been modelled and calibrated, to get an informative model that was then used for evaluating the potential of energy behaviour interventions in demand reduction. The work has used more than 200 sensors to ensure that the results were close to those in reality, and the model has been seen to represent the demands accurately. The savings obtained have been seen to be substantial, especially for winter where a poor mental model of occupants is making them select set point temperatures that are too high, even affecting negatively their comfort.
\end{abstract}

\section{Introduction}

Buildings are responsible for around $40 \%$ of the energy demand in developed countries (Pérez-Lombard, Ortiz, \& Pout, 2008). However, society has a great opportunity on reducing the emissions coming from that demand, as the sector has been seen to have a great potential of energy savings (Schnieders \& Hermelink, 2006). The scientific literature suggests that savings in energy of $20 \%$ can be achieved if interventions to change behaviour are carried out in buildings (Hargreaves, Nye, \& Burgess, 2010). The latest research has shown that when these behaviouralchange interventions are based in feedback to users that is in real time in a personalised fashion the savings are higher than when providing general feedback (Mogles, et al., 2017).

The new paradigm of IoT in buildings opens the opportunity to use sensors that are already installed in buildings or which marginal cost is rather low, to create a framework capable of delivering personalised real time feedback aimed to tackle the behaviours that represent the largest energy waste (Stankovic, 2014).

This has been done in project ENTROPY, resulting a framework that uses an IoT platform as the core to administer the data, create the logic that detects energy waste, elaborate messages (that are personal and scheduled), and deliver the information via created-forpurpose mobile apps. This paper shows the details about the framework and evaluates the performance of the activities carried out within it. The main objective of this project as part of the umbrella goals of the European Society is the reduction of energy use in building to eventually reduce Carbon emissions from buildings.

This work has been, to the knowledge of the authors, successful as it has proven that saving energy via intelligent feedback to building users is possible. The provided functional platform and a set of mobile phone applications could be a market product in the near future, specially directed to both public and private office buildings. These solutions have been seen to our perception to almost reach commercial maturity, and to provide added value to the European society because of the innovation they represent, and because of their effectiveness on saving energy.

\section{Methodology}

\section{The building}

The pilot building hosts the Faculty of Chemistry of the University of Murcia and it has already been used for energy consumption prediction experiments and smart city scenarios (González-Vidal, Ramallo-González, Terroso-Sáenz, \& Skarmeta, 2017) (Moreno, et al., 2017). Due to the nature of the faculty, the use of the building is very diverse. Among its uses one can find: lecturing, experimentation in labs, office use and storage.

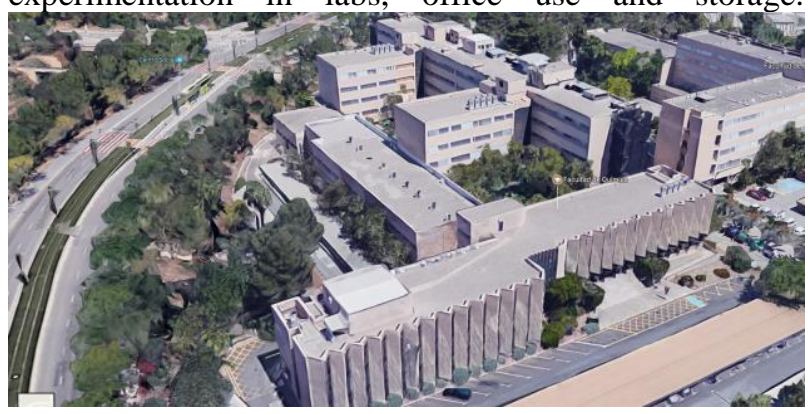

Figure 1: Bird view of the building from the West. 
The faculty has provided ENTROPY's team with a rather complete set blue prints of the building, what has facilitated the construction of an EnergyPlus model that contains all the zones of the building. However, it should be mentioned that other non-visible aspects of the building were not know and had to be chosen using national and international statistics.

The building is oriented to the South East. There is a nearby building in front of the South-East facade, that has been taken into consideration for projected shadows. The construction seems to be solid wall on a concrete structure. All windows are single glazing sliding windows with poor sealing. The frame of the windows is aluminium and it is seven centimetres wide in all parts of the frame, almost all situated on the inner part of the $40 \mathrm{~cm}$-thick walls, except for the windows on the sawshaped facade (see Figure 2).

The building is being monitored with the following:

- One electrical networks analyser at the building level,

- 230 Temperature sensors in selected zones,

- 230 Thermostat temperatures in selected zones,

- One external temperature sensor near the building site,

- One solar radiation sensor near the building site.

The gains of the building were difficult to calculate. With a building of such dimensions it would have been close to impossible to identify by inspection every electric equipment, also, some of the areas were restricted. To overcome this problem, the data available from the building level power meter was used to infer the electricity consumption. A further description on this will be given in following sections.

The total area of the building was $18250.9 \mathrm{~m} 2$ as simulated. This area is shared between five floors containing each one up to four different zones. The zones represent areas of the building containing several rooms. The model has a total of thirteen thermal zones, 345 surfaces (walls) and 683 windows. Internal thermal mass has been added to the model to ensure that the partitions separating rooms are represented in the thermodynamic equations.

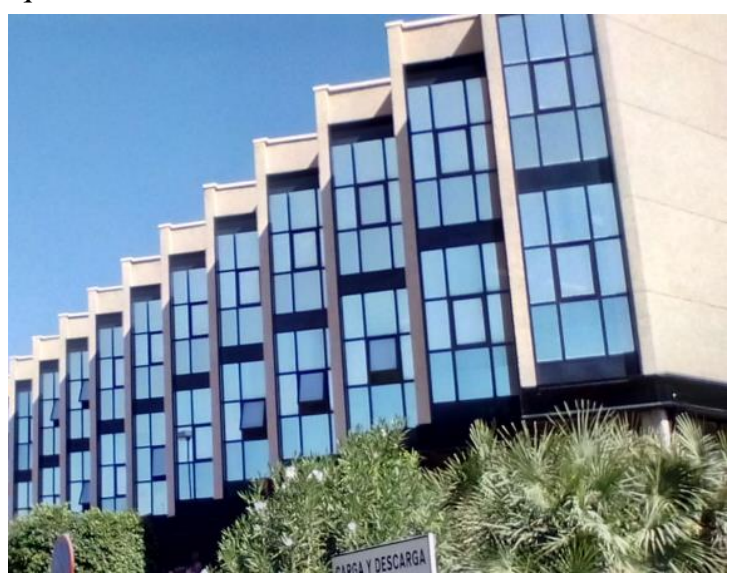

Figure 2: Detailed photo of the saw-shaped facade.
For the creation of the thermal model EnergyPlus was used. Due to the complexity and size of the building at hand, a mathematical software (Octave) was used to generate the geometry. With this approach, it was possible to create a parametric model, allowing to do further investigation of "what if" scenarios also in the geometry of the model. This allows to compare soft energy efficiency measurements (as the one on ENTROPY) with "hard" energy efficiency measurement such as changing windows, placing overhangs and so forth.

A representation of the geometry of the First floor of the building generated with Octave can be seen in Figure 3 .

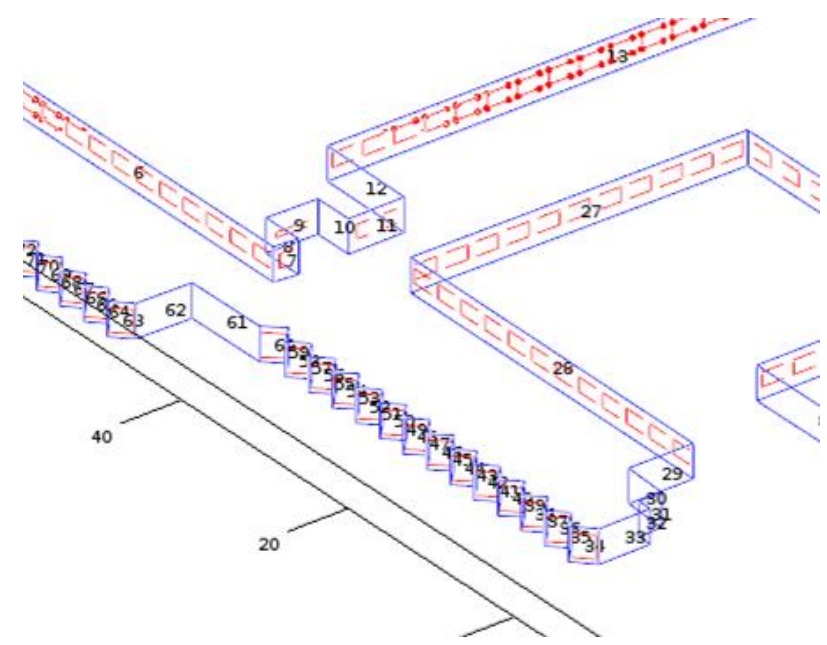

Figure 3: Detail of the parametric geometry generated with the mathematical software Octave.

One of the most significant characteristics of the building are its Venetian blinds on the windows of the South-East and South-West facades. A schematic representation of the parameters for blinds in EnergyPlus have been shown in Figure 4.

This type of blinds is highly effective to stop direct and most diffuse solar radiation and therefore reduce the cooling demand of the building in summer, and maximise the solar gains in winter and reduce the cooling demand. For this to occur, seasonal or even weekly management of the blinds has to be done. A quick visual inspection of the building is enough to verify that the slats are not oriented consistently, what suggests that not all of them are being optimally controlled. On the model we have selected a slat angle of 90 degrees.

To illustrate the effect of the vertical slats, an example has been created in which a box-like house has been modelled with two windows: One of the windows has no slats and the other one has vertical slats as the ones in the building of the Faculty of Chemistry. The result of this test can be seen on Figure 5.

As mentioned before the blinds on the model described in this document has been assigned the angle of 90 degrees as no consistency has been seen on those of the building under study. 


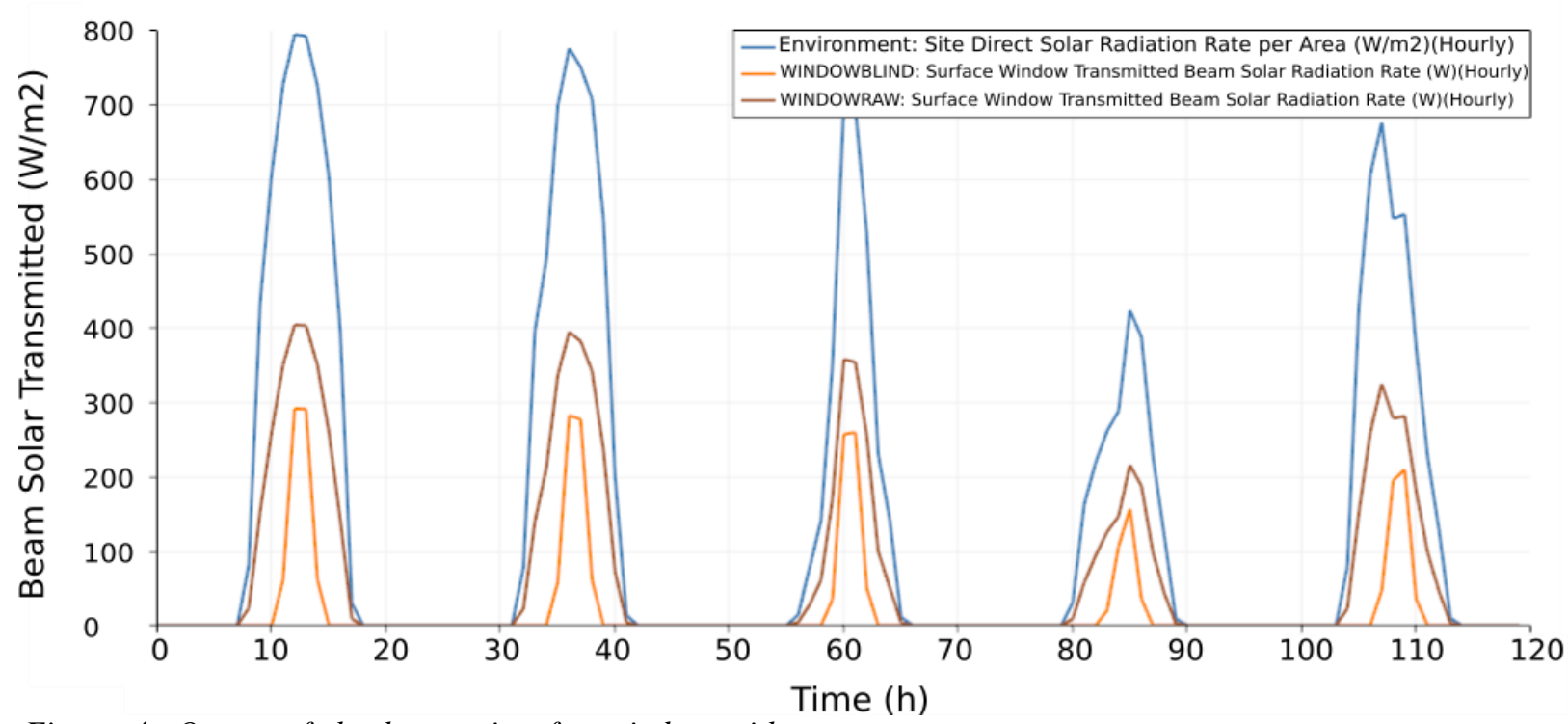

Figure 4: Output of the heat gain of a window with

Time (h)

Venetian blinds and its counterpart without them.

With respect to the conditioning system of the building, several units are present and functioning at the same time. The building is equipped with boilers that help heating up the air of the main ventilation system;

but in addition to that, Variable Refrigerant Flow (VRF) units are installed in all premises of the building to provide cooling.

The data from these VFR units is remotely accessible and it has been the main source of information for the modelling of the operation of the building.

\section{IoT infrastructure}

The IoT platform used in this work is described in detail on (Terroso-Saenz, González Vidal, P. RamalloGonzález, \& F. Skarmeta, 2017), and similar to that shown in (Rodríguez-Rodríguez, González Vidal, Ramallo González, \& Zamora, 2018) but an overview is presented here for contextualisation.

The core of this architecture is based on the FIWARE platform, a key initiative of the Future Internet PublicPrivate Partnership (PPP) to create a well-aligned set of

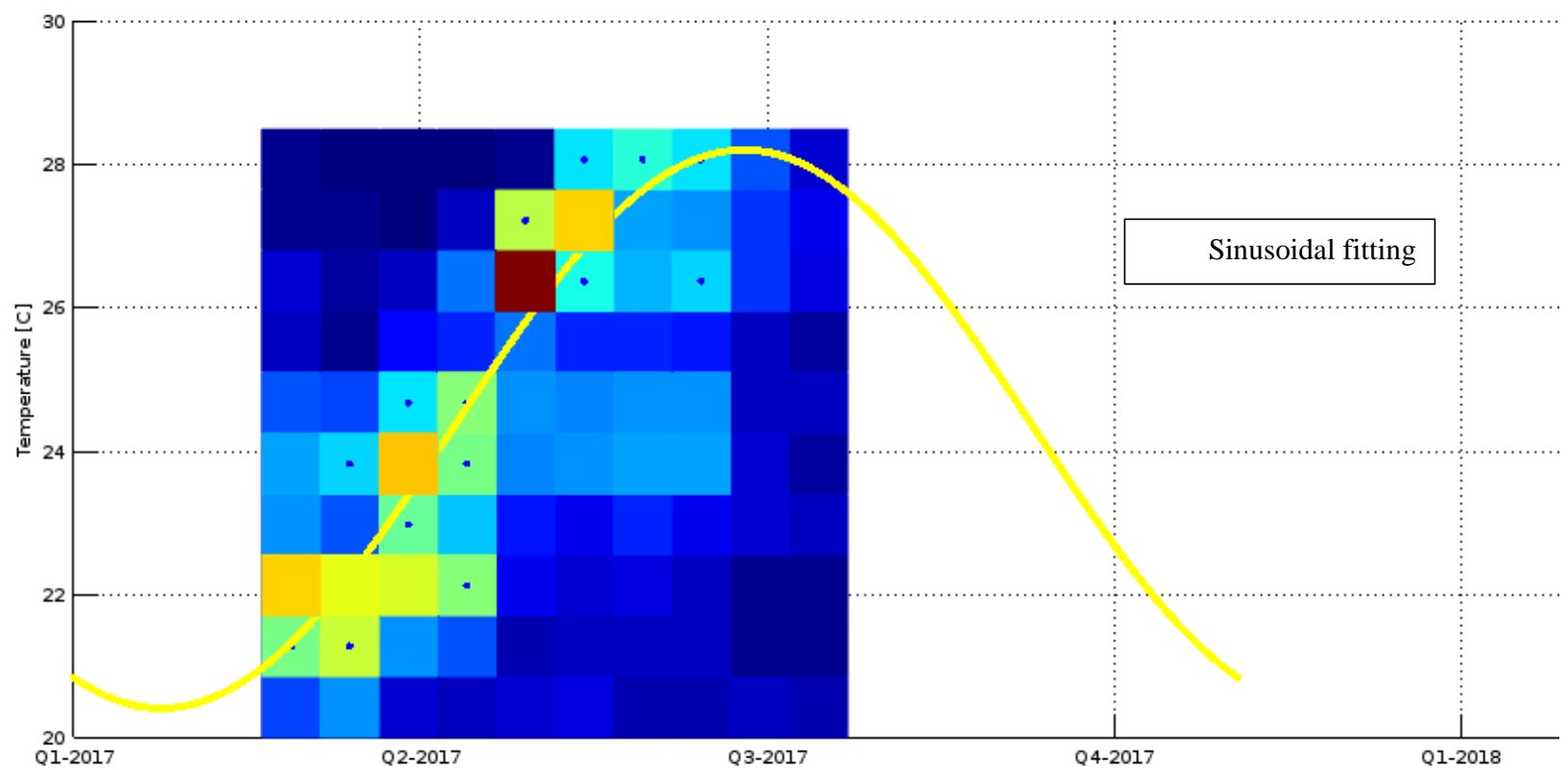

Figure 5: 2D histogram of the internal temperatures in the building. 
open enablers to receive, process, contextualize and publish IoT data from and for smart cities including from city-wide information to dwelling specific data.

The sensorisation layer is in charge of connecting physical devices or actuators that are going to provide data to the platform. Once this is done, it maps the collected data to the NGSI entities of the information model and sends the mapped information to the upper homogenization and storage layer. For the realization of this sensorisation layer, we have made use of the FIWARE IoT Agent enabler (FIWARE 2018a). In a nutshell, this enabler allows to automatically perform the aforementioned data mapping. Different types of this enabler support transport protocols to connect to the physical devices like MQTT or Lightweight M2M. More specifically, the connexion of the $\mathrm{A} / \mathrm{C}$ machines was done via a Modbus-Master. Each one of the cards inside the machine is connected to the master using a RS-485 and communicates using the protocol Modbus-RTU. The communication cards have a Modbus/RS-485 interface in which they are considered slave nodes. A unique direction has been assigned to each one of them. In addition, the units have an TCC-LINK interface to connect to the internal bus of the machine.

\section{Intervention}

The aim of an energy intervention is to develop an effective campaign that will reduce the energy consumption of the building via the adoption of more efficient habits by the building users.

A platform to provide feedback was created that is capable to communicated to the user via mobile apps. Captures of some of the applications created can be seen in Figure 6

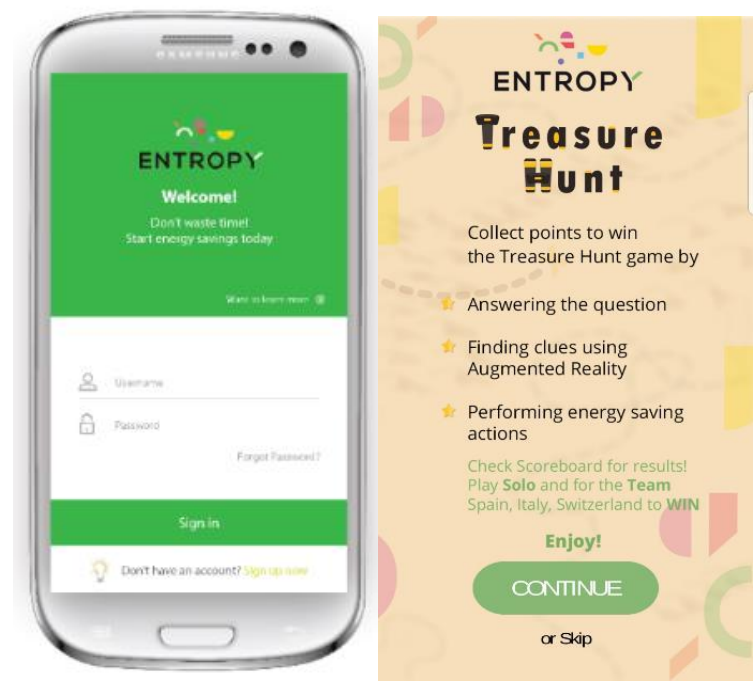

Figure 6: Screenshots of two of the apps developed in the project to provide feedback.

It is for this reason that it is crucial for this work to model the effect of occupants in this model properly. Only in this way, it will be possible to evaluate accurately the effect that changes on behaviour have over global consumption.

\section{Thermostat temperatures}

The internal temperature that a conditioning system has to maintain on a building is one of the single user-controlled parameters that have the largest effect on energy consumption. It is for this reason that thermostat temperature has been one of the target behaviours for the energy intervention of ENTROPY. The thermostat temperature of the year 2016 has been used to identify the values that are used before the intervention. This can be seen in Figure 7.

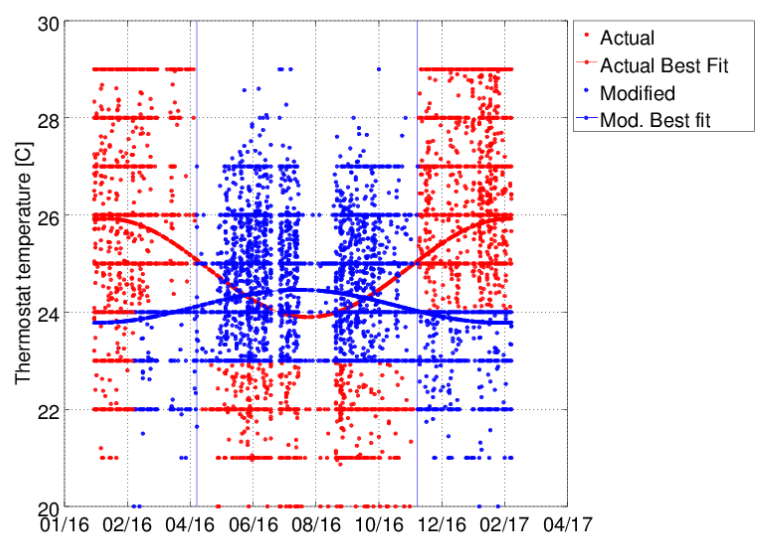

Figure 7: Information obtained from set point (thermostat) temperatures of 230 independent devices.

The thermostat temperature has been found to be inadequate in many cases, in summer and in winter. This proves that the energy consumption could be reduced substantially if a change on this behaviour is done.

Although this will be analysed in detail with the EnergyPlus model, it should also be mentioned here that a modified thermostat temperature time series has been created via the modification of temperatures that were seen inadequate to the upper or lower band (in winter and summer respectively) of the acceptable band i.e. 24 degrees in Winter and 23 degrees in Summer. Those temperature values that were below 24 in Winter or above 23 in Summer were not modified.

The two profiles that were used in EnergyPlus to analyse the change on thermostat behaviour can be seen on Figure 7. Their formulae are:

Tset current $=24.91-1.01 * \cos (2 * \mathrm{pi} / 365 *(\mathrm{t}+19.38))$;

Tset mod. $=24.12-0.339 * \cos (2 * \mathrm{pi} / 365 *(\mathrm{t}+210.7))$;

where tis the time in days. These formulae are the result of adjusting the real data of thermostat values over the year (red in Fig.8, Eq. 1) and the curve that will result when fitting the data once the set point temperatures that are out of the comfort range, and represent energy waste are corrected (blue in Fig.8, Eq. 2). It was interesting to see how the change on behaviour flips the shape of the 
curve to be concave on the summer, as one should expect considering the current theories of adaptive comfort.

\section{Internal Temperatures}

Although we had the thermostat values, we have also carried out a study on internal temperatures as the set point temperatures are not necessarily achieved on a real conditioning system. This has been proven after reevaluating the temperatures. One can see in Figure 6 that the internal temperatures found in the building do not resemble those of the set points. Nevertheless, we can still see that some change of behaviour would be beneficial. See for example rooms at 21 degrees in Summer. The machines that are monitoring the temperature are not capable to read temperatures below (and including) 19.5 or above (and including) 29 degrees. It is for this reason that we have to make an extrapolation of the values found to understand the real internal temperatures found in the building.

To start with, we fitted a sinusoidal curve to the data that was available, using the results of the histogram. We decided to use a sinusoidal curve, as the temperature

inside the building has to be cyclic and being this a response partly of the outside temperature it makes sense to be of the same shape. We used an optimisation algorithm to fit the amplitude and the phase of the curve using the data available (see Figure 1). From that, we obtained the following formula:

$$
\begin{gathered}
\mathrm{T}_{\text {in }}(\mathrm{t})=\mathrm{p} 1+\mathrm{p} 2 * \cos (2 * \mathrm{pi} / 365 *(\mathrm{x}+\mathrm{p} 3)) \\
\mathrm{P}_{1}=24.31 ; \mathrm{p} 2=-3.906 ; \mathrm{p} 3=211.50
\end{gathered}
$$

The most important aspect of this simulation was to evaluate how the change on the temperatures that are considered inadequate will result on a reduction of energy use. It is for this reason that the average internal temperature of the building was not enough. To calculate the dispersion of internal temperatures in the different rooms, we studied the standard deviation of the slices of the histogram shown in Figure 6. The following table shows the results of adjusting normal distributions to the slices of the two dimensional histogram. Due to the limited range of the readings some of the slices were incomplete, on those cases, the values were not considered on the fitting.

Table 1: Profiles calculations.

\begin{tabular}{|c|c|c|c|}
\hline & Mean & Sigma & Min Obj \\
\hline Slice 1 & 21.87 & 2.021 & $3.24 \mathrm{e} 6$ \\
\hline Slice 2 & 21.60 & 1.985 & $3.016 \mathrm{e} 6$ \\
\hline Slice 3 & 23.11 & 1.59 & $1.75 \mathrm{e} 6$ \\
\hline Slice 4 & 23.52 & 2.064 & $2.777 \mathrm{e} 6$ \\
\hline Slice 5 & 26.35 & 1.47 & $1.37 \mathrm{e} 7$ \\
\hline Slice 6 & 27.18 & 2.23 & $4.49 \mathrm{e} 6$ \\
\hline Slice 7 & 26.93 & 3.274 & $1.492 \mathrm{e} 6$ \\
\hline Slice 8 & 27.15 & 3.374 & $1.205 \mathrm{e} 6$ \\
\hline Slice 9 & 34.51 & 5.65 & $3.084 \mathrm{e} 5$ \\
\hline Slice 10 & 40.65 & 9.204 & $1.44 \mathrm{e} 5$ \\
\hline
\end{tabular}

After obtaining the standard deviation of the internal temperatures in each one of the slices of the histogram, we took the median as the true value of dispersion of internal temperature inside the building. Its value was 2.1 degree $\mathrm{C}$. With the median and the average internal temperature shown in Eq1, we were able to generate a synthetic internal temperature for the whole year. This served as input for EnergyPlus.

\section{Infiltration and ventilation}

Infiltration and ventilation are also big contributors to energy bills. Although infiltration cannot be controlled by the building users (window cracks, poor doors, etc.) ventilation can be controlled by opening and closing windows. The building at hand has all the windows openable, and a percentage close to $40 \%$ can be opened in each one of them. Leaving windows open while the heating or the cooling is on can result on a great deal of

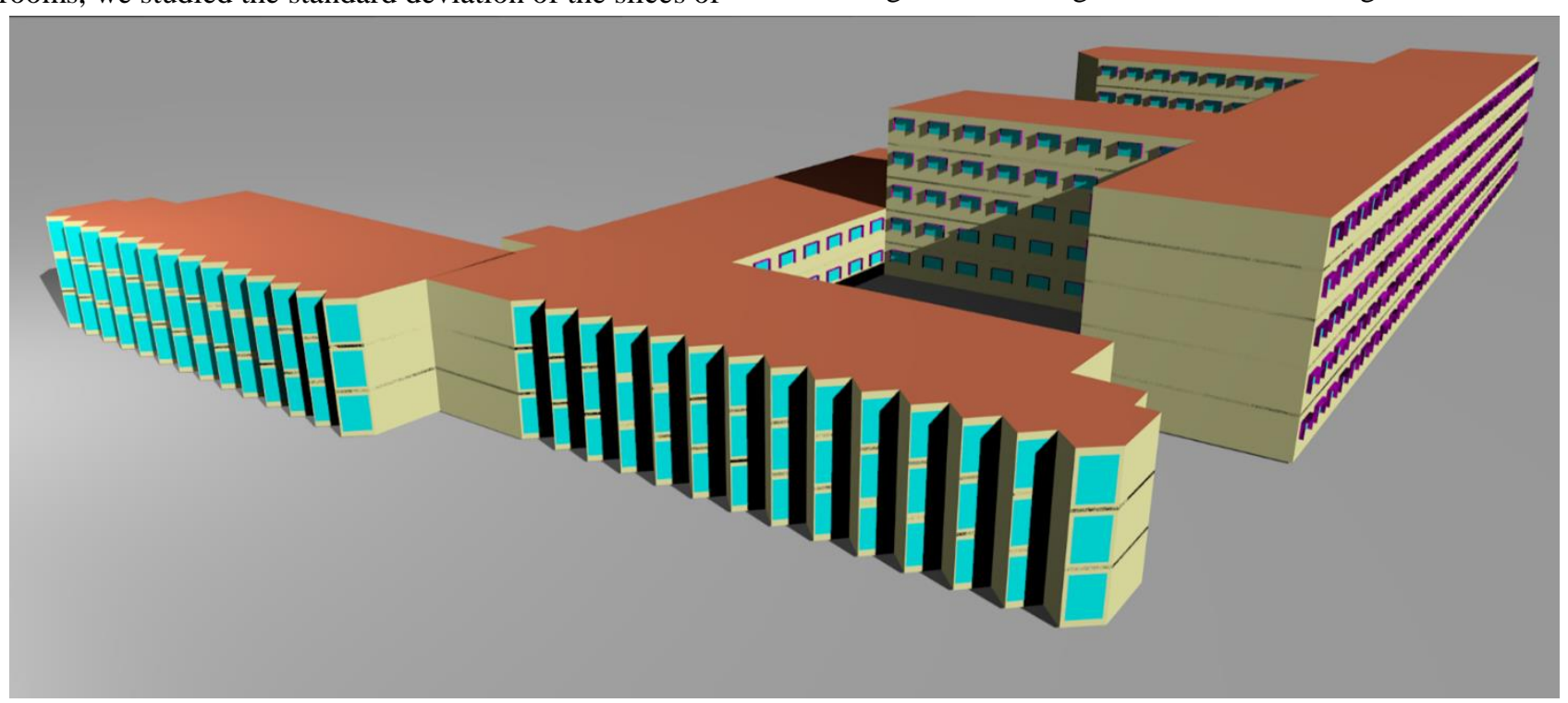

Figure 8: Rendering of the geometry of the EnergyPlus model. 


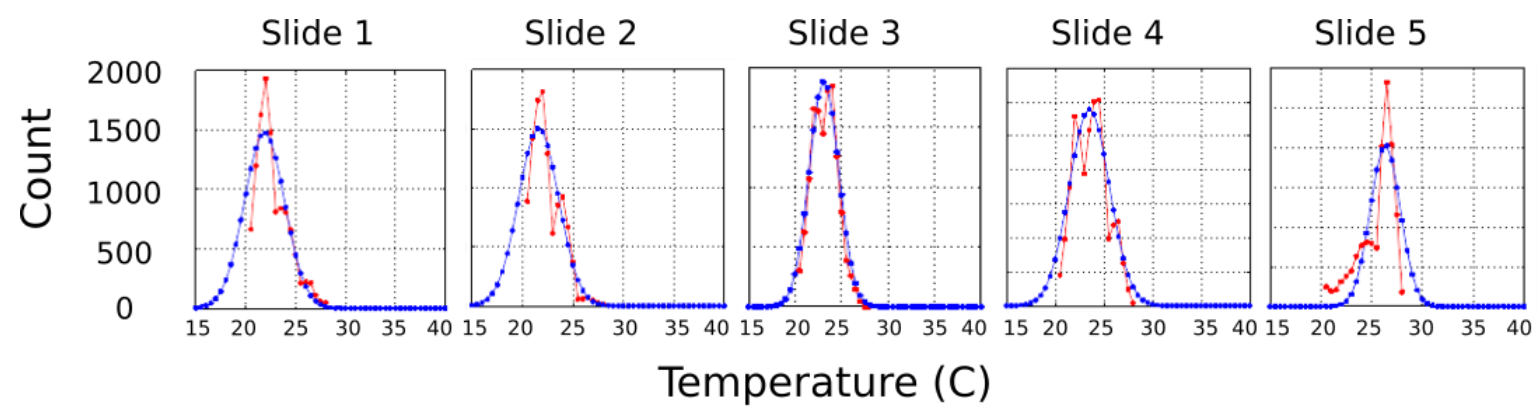

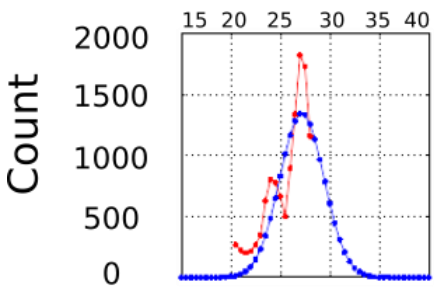

Slide 6

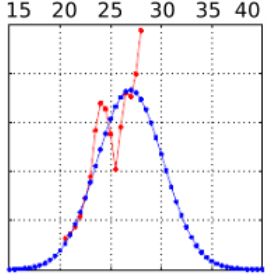

Slide 7

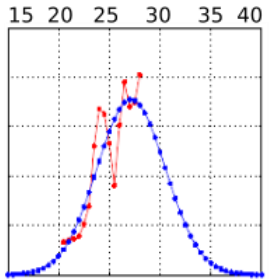

Slide 8

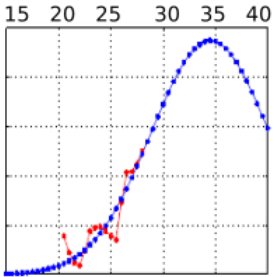

Slide 9

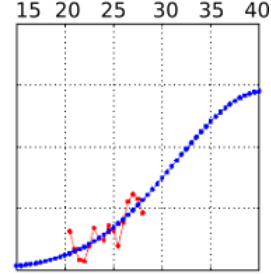

Slide 10

Figure 9: Profiles representations.

energy waste. This was also studied on the model. After the examination of the building we selected a rate of air renewal of $1 \mathrm{ach}$.

\section{Other gains}

Solar gains are automatically computed in the simulator, but electrical and metabolic gains have to be included in the input file. After evaluation of the electricity data gathered for the period of a year we concluded that the gains should be $2 \mathrm{~W} / \mathrm{m} 2$ in the case of lighting, $4 \mathrm{~W} / \mathrm{m} 2$ in the case of other electrical appliances and that the metabolic gains will correspond to 0.05 person $/ \mathrm{m} 2$.

\section{Simulation}

The simulation was performed over the building with the geometry generated after its inspection and using the gains and other inputs that have been described before.

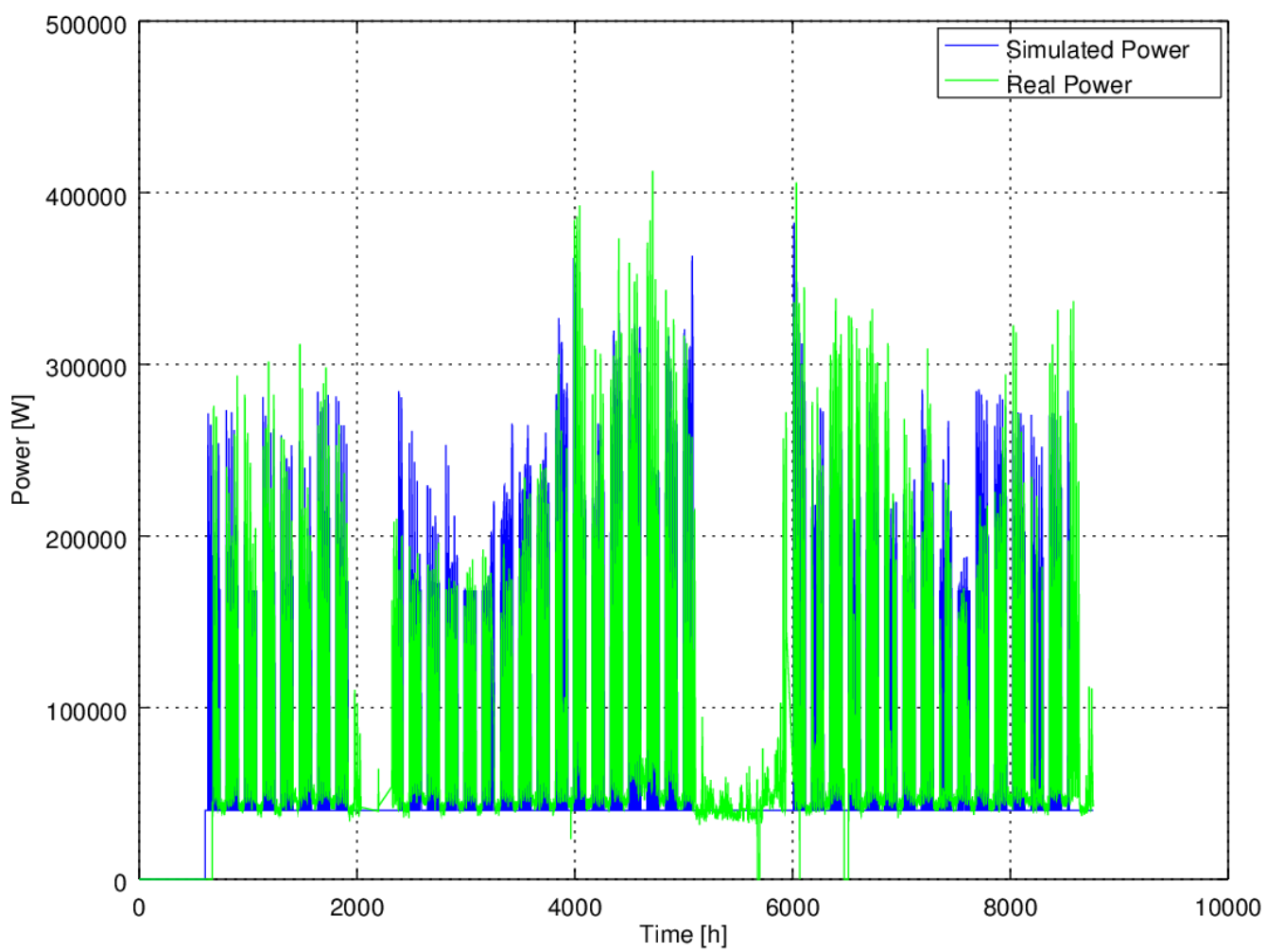

Figure 10. Results from the EnergyPlus model using the current status and operation of the building. 
The final model was rendered and is shown in Figure 9. The first simulation was done using all the inputs known from the building and its operation, trying to replicate the current situation. As there were values that were not know, fine running of the model was carried on to ensure that the outputs were as close as possible to the real data.

After obtaining this baseline, different scenarios were evaluated. Due to the large size of the model, and the large number of elements on it, each one of the simulations took a substantial amount of time, averaging around 32 minutes. Without extra tuning, and using the parameters expected for a building of this kind and this age, the

results were promising. The results of the simulation for the baseline can be seen in Figure 9.

After this, we have performed a series of simulations that provide with the energy consumption that would be expected for the interventions. The results of these can be seen on Table 1 . The evaluation was done considering that the heating set points were all moved to 21 degrees and not higher and that the cooling set points were set to 24 and not lower. Previous calculations were done to see the effect of making all heating set points not higher than 24, but the difference was not perceivable.

Table 2: Energy Demands.

\begin{tabular}{|c|r|r|r|}
\hline $\begin{array}{c}\text { Data in } \\
\text { MWh }\end{array}$ & \multicolumn{1}{|c|}{ Heating } & \multicolumn{1}{c|}{ Cooling } & \multicolumn{1}{c|}{ Lights } \\
\hline Real & n/a & n/a & n/a \\
\hline Baseline & 42.4 & 85.6 & 149.5 \\
\hline $\begin{array}{c}\text { Modified } \\
\text { Behaviour }\end{array}$ & 25.02 & 78.49 & 149.5 \\
\hline Savings \% & $41 \%$ & $8.3 \%$ & $0 \%$ \\
\hline
\end{tabular}

We saw from the simulation that an effective change on all behaviours would lead to a saving in the heating demands of $41 \%$ and a saving on cooling of $8.3 \%$. It is therefore clear that there is a great opportunity for reducing energy use by a change on behaviour, especially in winter.

Table 3: Energy Demands.

\begin{tabular}{|c|r|r|r|}
\hline $\begin{array}{c}\text { Data in } \\
\text { MWh }\end{array}$ & Op. Loads & \multicolumn{1}{c|}{$\begin{array}{c}\text { Residual } \\
\text { Loads }\end{array}$} & \multicolumn{1}{l|}{ Total } \\
\hline Real & n/a & n/a & 720 \\
\hline Baseline & 199.3 & 351.8 & 828 \\
\hline $\begin{array}{c}\text { Modified } \\
\text { Behaviour }\end{array}$ & 199.3 & 351.8 & 804 \\
\hline
\end{tabular}

\section{Conclusions}

The results of the model show that the modification of the behaviour of the building users can have a significant impact on the energy use of the building. The effect that changing the thermostat temperature has had was similar to that of changing the windows of the whole building although the former has very small cost compared to the later. Also, this model served to identify the gains of the model and to estimate the savings that could be achieved by the reduction of them in Summer. This could be done via the installation of overhangs or by the reduction of electrical gains using high efficiency devices. The study has shown that the electricity used in the building is substantial. It would be beneficial to promote the adoption of low-energy appliances and other devices and to ensure that the number of parasitic loads connected to the electrical circuit is minimal.

Overall we have seen that the change on behaviour can have a substantial effect on the energy consumption on the building that has been studied. The change on the thermostat values has been seen to be always positive and to have a substantial effect, producing savings on heating of $41 \%$ and on cooling of $8 \%$. As the overall electric load of the building is substantial, it is also recommended to lower the parasitic loads to have an even greater effect on the reduction of energy use.

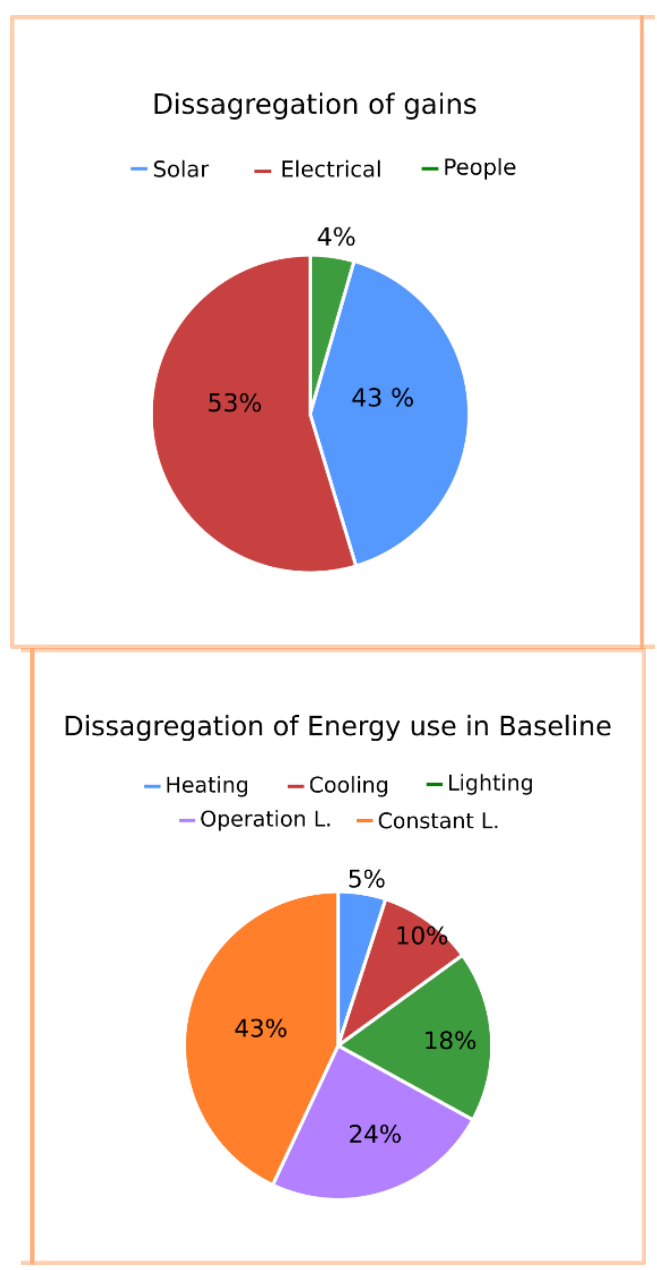

Figure 11:The model allowed to perform an approximate disaggregation of the demands what was key for designing the intervention.

The work here presented is a detailed study on how using publically available software one can calculate on a rather accurate way the savings that one may expect when performing an intervention to reduce energy use. It has been seen that although there is a great deal of uncertainties with respect to building characteristics, one can get a model that is rather accurate in parameters that affect the thermal comfort such as internal temperature, and also in parameters that are relevant for the total energy 
consumption i.e. electricity use for heating, cooling, lights and devices.

It has been seen in this work that it is highly relevant to have proxies of the energy behaviour that is happening in the building prior intervention. In our case, PIR sensors for presence and accurate internal temperature measurements to identify set point preferences have been key information to have a good impression of the use of the building. In the absence of these, the figures of the potential savings could have been misleading as we have identified that the energy consumption was rather different that one may expect from design (in accordance with the literature about the performance gap).

Overall, we have considered this work highly relevant for the community that aims at performing behavioural interventions to reduce energy use. This is an option that is becoming more and more popular with the popularisation of the Internet of Things and the new European directive that suggest that buildings should be Smart Ready. With smart meters and sensors already in place in building, these interventions will have a cost that can be considered negligible, but yet the savings can be as high as some of the hard interventions that one can do in a building.

\section{Acknowledgement}

This work has been sponsored by the Spanish Ministry of Economy and Competitiveness through PERSEIDES (ref. TIN2017-86885-R) and CHIST-ERA (ref. PCIN2016-010) projects and by MINECO grant BES-2015071956 and by the European Commission through the H2020 IoTCrawler (contract 779852) and DEMETER (grant agreement 857202) EU project.s RamalloGonzález thanks the program Saavedra Fajardo (grant number220035/SF/16) funded by CARM - Fundación Séneca-Agencia de Ciencia y Tecnología.

\section{References}

González-Vidal, A., Ramallo-González, A. P., TerrosoSáenz, F., \& Skarmeta, A. (2017). Data driven modeling for energy consumption prediction in smart buildings. Big Data (Big Data), 2017 IEEE International Conference on, (págs. 45624569).

Hargreaves, T., Nye, M., \& Burgess, J. (2010). Making energy visible: A qualitative field study of how householders interact with feedback from smart energy monitors. Energy Policy, 38, 6111-6119. doi:http://dx.doi.org/10.1016/j.enpol.2010.05.0 68

IDAE. (2009, 5). CALENER-GT: Grandes Edificios Terciarios. Manual Técnico. Madrid.
ISO. (2005). Ergonomics of the thermal environment -Analytical determination and interpretation of thermal comfort using calculation of the PMV and PPD indices and local thermal comfort criteria. ISO/TC 159/SC 5 , ISO.

Liu, B. Y., \& Jordan, R. C. (1960). The interrelationship and characteristic distribution of direct, diffuse and total solar radiation. Solar Energy, 4, 1-19. doi:https://doi.org/10.1016/0038092X(60)90062-1

Mogles, N., Walker, I., Ramallo-González, A. P., Lee, J., Natarajan, S., Padget, J., . . Coley, D. (2017). How smart do smart meters need to be? Building and Environment, 125, 439-450. doi:https://doi.org/10.1016/j.buildenv.2017.09.0 08

Moreno, M. V., Terroso-Sáenz, F., González-Vidal, A., Valdés-Vela, M., Skarmeta, A. F., Zamora, M. A., \& Chang, V. (2017). Applicability of big data techniques to smart cities deployments. IEEE Transactions on Industrial Informatics, 13, 800809.

Pérez-Lombard, L., Ortiz, J., \& Pout, C. (2008). A review on buildings energy consumption information. Energy and Buildings, 40, 394-398. doi:https://doi.org/10.1016/j.enbuild.2007.03.00 7

Robin Mitchell, Arasteh, D., Carmody, J., Huizenga, C., \& Curcija, D. (2003, 6). THERM 5 / WINDOW 5 NFRC simulation manua. University of California.

Rodríguez-Rodríguez, I., González Vidal, A., Ramallo González, A. P., \& Zamora, M. Á. (2018). Commissioning of the Controlled and Automatized Testing Facility for Human Behavior and Control (CASITA). Sensors, 18. doi:10.3390/s18092829

Schnieders, J., \& Hermelink, A. (2006). CEPHEUS results: measurements and occupants' satisfaction provide evidence for Passive Houses being an option for sustainable building. Energy Policy, 34, 151-171. doi:https://doi.org/10.1016/j.enpol.2004.08.049

Stankovic, J. A. $(2014,2)$. Research Directions for the Internet of Things. IEEE Internet of Things Journal, $1, \quad 3-9$. doi:10.1109/JIOT.2014.2312291

Terroso-Saenz, F., González Vidal, A., P. RamalloGonzález, A., \& F. Skarmeta, A. (9 de 2017). An open IoT platform for the management and analysis of energy data. 